Disclosure of Interests: Carina Borst: None declared, Farideh Alasti: None declared, Daniel Aletaha Grant/research support from: AbbVie, BristolMyers Squibb, and MSD, Consultant for: AbbVie, Bristol-Myers Squibb, Eli Lilly, Janssen, Medac, Merck, MSD, Pfizer Inc, Roche, and UCB, Speakers bureau: AbbVie, Bristol-Myers Squibb, Eli Lilly, Janssen, Medac, Merck, MSD, Pfizer Inc, Roche, and UCB

DOI: 10.1136/annrheumdis-2019-eular.2353

\section{FRI0666 RHEUMATIC COMPLICATIONS OF IMMUNE CHECKPOINT INHIBITOR THERAPY: A CASE SERIES}

Benedict Moore ${ }^{1}$, Matthew $\mathrm{Ho}^{2}$, Margaret Callan ${ }^{2} .{ }^{1}$ Imperial College School of Medicine, London, United Kingdom; ${ }^{2}$ Chelsea and Westminster Hospital, London, United Kingdom

Background: Immune checkpoint inhibitor therapy reduces negative sig nals of $T$ cell activation and enables tumour-specific $T$ cells to mount a more effective response. Use of these drugs in management of malig nancy has been associated with the development of auto-immune disease. There are few published studies describing immune-related adverse events affecting the musculoskeletal system.

Objectives: This study aimed to describe the spectrum of musculoskeletal presentations in patients treated with programme death (PD)-1 and/or cytotoxic $T$ lymphocyte associated antigen (CTLA)-4 blockade.

Methods: 16 patients were identified by retrospective review of records. All patients had received treatment with monoclonal antibodies specific for PD-1, PD ligand-1 and/or CTLA4 and had been referred for rheumatology assessment of musculoskeletal complications. We assessed clinical presentation, results of blood tests for C-reactive protein (CRP), anti-nuclear antibody (ANA), rheumatoid factor (RF) and anti-cyclic citrullinated peptide (CCP) antibody, and response to treatment.

Results: Seven males and nine females, with mean age of 56 years were evaluated. 13 patients had melanoma, two renal cell cancer and one triple negative breast cancer. Patients presented with a spectrum of musculoskeletal symptoms. Five patients displayed features most consistent with polymyalgia rheumatica (PMR), five had peripheral spondyloarthritis (SPA) features with lower limb oligoarthritis or plantar fasciitis, four had rheumatoid arthritis (RA) features with small joint polyarthritis, one had myositis with raised creatine kinase and one had new onset of gout. Only one of 16 patients was positive for RF. All patients were seronegative for anti-CCP antibodies and ANA. One patient with RA presentation had a high CRP of $39 \mathrm{mg} / \mathrm{L}$. Five patients had a mildly elevated CRP of $5-10 \mathrm{mg} / \mathrm{L}$. Other patients did not show an acute phase response. All patients received treatment with corticosteroid with benefit. Intra-articular and soft-tissue corticosteroid injections were also effective. One of two patients with RA presentation responded to sulfasalazine, and none of two patients with RA presentation responded to methotrexate. One patient with RA presentation and one with SPA presentation responded to tumour necrosis factor (TNF)alpha blockade.

Conclusion: Attenuating inhibitory signals of $T$ cell activation using immune checkpoint inhibitor therapy is associated with a range of rheumatic complications, including PMR-like as well as RA- and peripheral SPA-like presentations. The majority of patients were seronegative for RF, CCP and ANA, and had low or borderline elevated CRP. All patients showed a response to prednisolone. TNF-alpha blockade was effective for RA and SPA presentations.

\section{REFERENCES:}

[1] Mitchell EL, Lau PKH, Khoo C, et al. Rheumatic immune-related adverse events secondary to anti-programme death- 1 antibodies and preliminary analysis of the impact of corticosteroids on anti-tumour response: A case series. Eur J Cancer 2018; 105:88-102.

[2] Richter MD, Crowson C, Kottschade LA, et al. Rheumatic syndromes associated with immune checkpoint inhibitors: a single centre cohort of 61 patients. Arthritis Rheumatol 2018 doi:10.1002/art.40745.

[3] Mooradian MJ, Nasrallah M, Gainor JF, et al. Musculoskeletal rheumatic complications of immune checkpoint inhibitor therapy: A single center experience. Semin Arthritis Rheum 2018 Doi: :10.1016/j. semarthrit.2018.10.012.

Disclosure of Interests: None declared DOI: 10.1136/annrheumdis-2019-eular.2397

\section{FRI0667 \\ IS OBESITY A FACTOR OF POOR OUTCOME IN SPONDYLOARTHRITIS? AN ANALYSIS OF 638 PATIENTS}

Pascal Claudepierre ${ }^{1}$, Laure Gossec ${ }^{2,3}$, Laurent Grange ${ }^{4,5}$, Marco GarridoCumbrera $^{6,7,8}$, Emilie Desfleurs ${ }^{9}$, Francoise Alliot Launois ${ }^{10}$, René-Marc Flipo ${ }^{11,12}$. ${ }^{1}$ Henri Mondor University Hospital, Rheumatology, Créteil, France; ${ }^{2}$ Sorbonne University, Paris, France; ${ }^{3}$ La Pitié-Salpêtrière University Hospital, Rheumatology, Paris, France; ${ }^{4}$ Grenoble Alpes University Hospital, Rheumatology, Echirolles, France; ${ }^{5}$ Association Française de Lutte Antirhumatismale A.F.L.A.R, President, Paris, France; ${ }^{6}$ Universidad de Sevilla, Sevilla, Spain; ${ }^{7}$ Health and Territory Research (HTR), Sevilla, Spain; ${ }^{8}$ Spanish Federation of Spondyloarthritis Associations (CEADE), Madrid, Spain; ${ }^{9}$ Novartis Pharma, Medical Affairs, Rueil Malmaison, France; ${ }^{10}$ Association Française de Lutte Antirhumatismale A.F.L.A.R, Vice-President, Paris, France; ${ }^{11}$ Lille University, Lille, France; ${ }^{12}$ Roger Salengro University Hospital, Rheumatology, Lille, France

Background: There are few data on obese patients with axial spondyloarthritis (axSpA)

Objectives: To explore the impact of body mass index (BMI) on per sonal, professional and social life of axSpA patients.

Methods: BetweenDecember 2017 and February 2018, French patients followed for axSpA by their rheumatologists or affiliated to the French patients association AFLAR, and self-reporting axSpA, participated in the European Map of Axial Spondyloarthritis (EMAS) cross-sectional patient survey ${ }^{1}$. Sociodemographic data (including weight and height), axSpA characteristics and disease impact on personal (social interactions, frequency of social activities) and professional life (working hours, sick leave, and disability) were collected via an online questionnaire. Patients were classified in two groups according to their BMl (obese $\mathrm{BMI} \geq 30 \mathrm{~kg} / \mathrm{m}^{2}$, non-obese: $\mathrm{BMl}<30 \mathrm{~kg} / \mathrm{m}^{2}$ ) and a comparison between the two groups was conducted using chi2 or Mann-Whitney tests. There was no imputation of missing data.

Results: Data of 638 patients, mainly women 77\%, median age (years) $41.5 \pm 11.1$ were collected in France in 2018. Median BMI was $26.1 \pm 5.5$ and $22.1 \%$ patients were obese $(n=141)$. Median age was significantly higher in obese patients $(44.1 \pm 9.9$ vs $40.8 \pm 11.3 ; p=0.001)$, but there was no difference regarding gender, level of education and socio-professional categories. The obese had a longer diagnosis delay for axSPA (median $8.2 \pm 8.4$ vs $6.6 \pm 8.1$ years; $p=0.01$ ). They were more to report psoriasis $(33.8 \%$ vs $21.9 \% ; \quad p=0,005)$ and the following comorbidities: anxiety depression fibromyalgia, high blood pressure, hypercholesterolemia, diabetes and renal failure. Disease activity was also higher in the obese population: median BASDAI $6.5 \pm 1.5$ vs $5.8 \pm 1.8(p<0.001)$ and BASDAI $\geq 4$ in $92.9 \%$ vs $84.3 \% \quad(p<0.01)$. The same proportion of patients $(61.9 \%)$ has been treated by a biologic in the two groups. The impact of axSPA on personal life was more frequently reported by the obeses: $65.2 \%$ vs $48.0 \% \quad(p<0.005)$ had reduced the frequency of sport activities because of axSpA and $42.5 \%$ vs $28.3 \% \quad(\mathrm{p}<0.005)$ their sexual intercourse. There were a lower number of obeses, who had a regular physical activity (58.9\% vs 68.8\%; p>0.05) with a shorter mean duration of sport practice At the opposite, no difference was observed regarding the impact of axSpA on professional life, with a similar proportion of obese and nonobese patients reporting sick leave $(32.1 \%$ vs $24.9 \%)$ or disability $(31.9 \%$ vs $28 \%$ ). The mean number of working hours for the active people was also similar (35.5 vs 34.2 hours) in the two groups.

Conclusion: In this survey, obesity affected $22.1 \%$ of axSpA patients and was associated to a higher disease activity and a greater impact on personal life of axSpA, but not on their professional life. Obesity and its consequences have to be considered in the management of axSpA as it is already the case for psoriatic arthritis.

\section{REFERENCES:}

[1] Gossec, et al. Diagnostic Delay and Associated Factors in Axial Spondyloarthritis across Europe. Results from the European Map of Axial Spondyloarthritis Survey. Arthritis Rheumatol. 2018 Sep;70 Suppl 9:688

Acknowledgement: Survey supported by Novartis

Disclosure of Interests: Pascal Claudepierre Consultant for: Honoraria from Novartis as steering committe of this survey, Laure Gossec Grant research support from: AbbVie, BMS, Celgene, Janssen, Lilly, MSD Novartis-Sandoz, Pfizer, Sanofi, and UCB, Consultant for: AbbVie, Biogen, BMS, Celgene, Janssen, Lilly, MSD, Nordic Pharma, Novartis-Sandoz, Pfizer, Roche, Sanofi, and UCB, Consultant for: L Gossec has received honoraria from Celgene as investigator for this study, LAURENT GRANGE Consultant for: Laurent Grange has received honoraria from Amgen, Lilly and UCB and research support from Lilly, Amgen, UCB Expanscience, Mylan, Roche diagnostics and TEVA, Marco Garrido-Cumbrera Consultant for: Honoraria from Novartis as steering committe of this survey, Emilie Desfleurs Employee of: Employee of Novartis Pharma 
Francoise Alliot Launois: None declared, René-Marc Flipo Consultant for: Honoraria from Novartis as steering committe of this survey DOI: 10.1136/annrheumdis-2019-eular.2775

\section{FRI0668 FIRST DATA OF THE TARDIS-RAREGISTRY, A NATIONWIDE BELGIAN BIOLOGIC REGISTRY}

Diederik De Cock ${ }^{1}$, Patrick Durez ${ }^{2,3}$, Dirk Elewaut ${ }^{4,5}$, Bernard Lauwerys ${ }^{2,3}$ Rene Westhovens ${ }^{1,6}$, Patrick Verschueren ${ }^{1,6}$, Royal Belgian Society for Rheumatology. ${ }^{1} \mathrm{KU}$ Leuven, Skeletal Biology and Engineering Research Centre, Leuven, Belgium; ${ }^{2}$ Université Catholique de Louvain, Pôle de Pathologies Rhumatismales Inflammatoires et Systémiques, Institut de Recherche Expérimentale et Clinique, Brussels, Belgium; ${ }^{3}$ Cliniques Universitaires Saint-Luc, Department of Rheumatology, Brussels, Belgium; ${ }^{4}$ Ghent University, VIB Center for Inflammation Research, Ghent, Belgium; ${ }^{5}$ Ghent University Hospital, Department of Rheumatology - MRB2, Ghent, Belgium; ${ }^{6}$ University Hospitals Leuven, Department of Rheumatology, Leuven, Belgium

Background: The Tool for Administrative Reimbursement Drug Information Sharing (TARDIS) is an electronic platform combining data collection from patients with Rheumatoid Arthritis (RA) on biologic and synthetic targeted therapy, together with the submission of a request for reimbursement of this medication.

Objectives: To present the first data ever of the TARDIS-RA registry concerning the use of biologic and synthetic targeted therapy across Belgium. Methods: The Belgian TARDIS-RA registry started in April 2015. All Belgian rheumatologist were obliged after a transition period to insert patient data via the online portal when prescribing. If data of a patient are entered for the first time, previous and current use of classical synthetic (cs), targeted synthetic (ts) and biologic (b) disease modifying antirheumatic drug (DMARD) therapies is registered. Afterwards, every next bDMARD or tsDMARD initiation, prolongation and discontinuation is registered electronically in the system. Other data captured are basic demographic information such as age, gender and disease duration and disease characteristics including ESR, CRP, joint counts, disease activity scores and $\mathrm{HAQ}$.

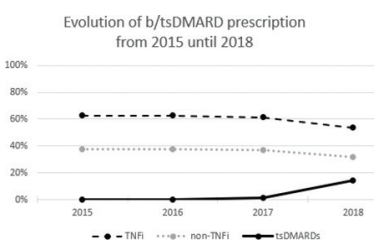

Results: At 18/01/2019, information on 80098 drug prescriptions in 13 614 patients was collected in the TARDIS-RA registry. Of these 80098 drug prescriptions, $30434(38.0 \%)$ were csDMARDs and glucocorticoids, and $49664(62.0 \%)$ were bDMARD or tsDMARD prescriptions. This last category consisted of $30380(61.2 \%)$ tumour necrosis factor inhibitor (TNFi) bDMARDs, 17104 (34.5\%) non-TNFi bDMARDs and 2170 $(4.3 \%)$ tsDMARDs. Only $642(2.3 \%)$ TNFi biosimilar prescriptions were registered. Fig1 shows the yearly evolution of bDMARD and tsDMARD prescription in Belgium from 2015 to 2018.

At first registered interaction with the rheumatologist, the 13614 patients had a median (IQR) age of 59 (50-68) years with a median (IQR) disease duration of 9 (4-16) years; $9666(73.4 \%)$ were female. The clinical characteristics showed a median (IQR) DAS28CRP of $3.6(2.1-4.8)$ and a median (IQR) HAQ of 1.1 (0.6-1.8). Of patients at first time registration, $37.6 \%$ could be validated as being bionaïve and $62.5 \%$ as bioexperienced. Conclusion: Approximately 70000 individuals in Belgium are estimated to have a RA diagnosis. Corresponding to numbers of other western European countries, $20 \%-25 \%$ of them would be expected to receive ts/ bDMARDs. This suggests that almost the entire Belgian RA population on biologic treatment is covered by TARDIS. The registration of initiation, prolongation and discontinuation of every ts/bDMARD since 2015 combined with the simultaneous collection of demographic and clinical data, will make the TARDIS-RA registry a useful and powerful tool for the long-term drug analyses in Belgian patients with RA.

Disclosure of Interests: Diederik De Cock: None declared, Patrick Durez Speakers bureau: Bristol-Myers Squibb, Eli Lilly, Sanofi, Celltrion, Dirk Elewaut: None declared, Bernard Lauwerys: None declared, Rene Westhovens Grant/research support from: Bristol-Myers Squibb, Consultant for: Celltrion, Galapagos-Gilead, Patrick Verschueren Grant/research support from: Unrestricted Pfizer Grant for Early RA research
DOI: 10.1136/annrheumdis-2019-eular.4747

\section{FRI0669 DEPRESSION AND ANXIETY AND THEIR CLINICAL CORRELATES IN SYSTEMIC LUPUS ERYTHEMATOSUS (SLE)}

David Eldeiry ${ }^{1}$, Moe Zandy ${ }^{2}$, Oshrat Tayer-Shifman ${ }^{2}$, Sherief Marzouk ${ }^{3}$, Jiandong Su', Zahi Touma ${ }^{2}{ }^{1}$ Royal College of Surgeons in Ireland, Medicine, Dublin, Ireland; ${ }^{2}$ University of Toronto, Rheumatology, Toronto, Canada; ${ }^{3}$ University of Toronto, Psychiatry, Toronto, Canada

Background: In our recent systematic review, we have shown a high prevalence for depression [35\% (95\% Cl: 29.9\%-40.3\%)] and anxiety [25.8\% (95\% Cl: 19.2\%-32.9\%)] in Systemic Lupus Erythematosus (SLE) ${ }^{1}$. A better understanding of the SLE phenotypic manifestations associated with depression and anxiety may lead to enhanced early diagnosis and treatment strategies.

Objectives: To determine the overall prevalence of anxiety and depression in a cohort of SLE patients, stratified by SLE-implicated organ sys tems and to study their correlates.

Methods: Patients attending the Toronto Lupus Clinic from August 2017 to January 2019 were studied. Depression and Anxiety were diagnosed with Center for Epidemiological Studies-Depression Scale (CES-D; cutoff $\geq 26$ ), BECK Depression Inventory-II (BDI-II; cut-off $\geq 18$ ), and the BECK Anxiety Inventory (BAI; cut-off $\geq 19$ ). Disease activity was measured with the SLEDAI-2k (SLE Disease Activity Index 2000). The SLE phenotypic manifestations were stratified based on the organ systems of cumulative 10-year SLEDAI-2K - skin, musculoskeletal (MSK), ocular, neuropsychiatric, and internal organ manifestations (including renal, pulmonary, immunologic, and hematologic). Separate univariate and multivariate logistic regression analyses (for depression [D], anxiety $[A]$, and anxiety and depression $[A D]$ ) were performed to study the factors associated with $A$, $D$, and $A D$, including age at enrollment, sex, ethnicity, disease duration, inception status (enrolled in the clinic within 1 year of SLE diagnosis), fibromyalgia, and SLE phenotypic manifestations, comparing their significance to the group with neither A or D.

Results: 341 patients $(89.7 \%$ female), with mean age $45.9 \pm 17.8$ years were studied. The prevalence of anxiety and depression in the cohor was $34 \%$ and $27 \%$ respectively, while $21 \%$ of the total cohort was found to have both anxiety and depression. Among the 3 outcome groups ( $A$, $D$, and $A D)$, MSK system involvement has a significantly higher prevalence when compared to the group with neither $A$ or $D$ ( $p$-values<0.05) Concurrently, skin system involvement was also significantly more prevalent among $A, D$, and $A D$ groups, in comparison to the normal group ( $p$ values $<0.05)$. Patients with anxiety had significantly higher odds of skin system involvement compared to the normal group $(\mathrm{OR}=1.81 ; 95 \% \mathrm{Cl}$ : $1.09,3.01)$. Also, patients with depression had higher odds of MSK $(\mathrm{OR}=1.94 ; 95 \% \mathrm{Cl}: 1.07,3.50)$ and skin system involvement $(\mathrm{OR}=1.79$ $95 \% \mathrm{Cl}: 1.00,3.20)$ compared to the group with neither A or D. Additionally, the odds of skin system involvement was significantly higher among patients with both $\mathrm{A}$ and $\mathrm{D}$, compared to the group with neither $(\mathrm{OR}=2.02,95 \% \mathrm{Cl}: 1.05,3.88)$. In all three models $(A, D$, and $A D)$, employment and fibromyalgia were also significant $(p$-values $<0.05)$. Age at enrolment was significant in the $D$ and $A D$ models ( $p$-value $<0.05$ ), while inception patient status [inception had a mean disease duration at study visit of $12.1 \pm 11.4$ years compared to non-inception $19.6 \pm 10.6$ years] was significant in the D model exclusively ( $p$-value $<0.05$ ).

Conclusion: SLE phenotypic manifestation, specifically those involving patients' skin or MSK systems, along with fibromyalgia, socio-demographic factors, and inception status were associated with anxiety or depression. Routine patient screening and evaluation, especially among patients with shorter disease duration, for these correlates may facilitate the diagnosis of these mental health disorders, and allow for more timely diagnosis and intervention strategies.

\section{REFERENCES :}

[1] Moustafa A, Hassanein M, Eder L, Wither JE, Fung W, Lambiris P, Touma Z. Prevalence and Metric of Depression and Anxiety in Lupus: A Systematic Review and Meta-Analyses [abstract]. Arthritis Rheumatol. 2016; 68 (suppl 10).

Disclosure of Interests: David Eldeiry: None declared, Moe Zandy: None declared, Oshrat Tayer-Shifman: None declared, Sherief Marzouk: None declared, Jiandong Su: None declared, Zahi Touma Grant/research support from: GSK Canada, Consultant for: UBC, Pfizer, Janssen, Inc DOI: 10.1136/annrheumdis-2019-eular.7595 